\title{
Association Between Vitamin D Deficiency and Duration of Hospital Stay, Pediatric Intensive Care Unit Stay, and Ventilation; Pediatric Risk of Mortality Score; and Rate of Readmission: A Prospective Observational Study
}

\author{
Sarthak Das ${ }^{1}$, Kiran Kumar $\mathrm{M}^{2}$, Niranjan Biswal ${ }^{2}$, Narayanan Parameswaran ${ }^{2}$, Nivedita Nanda ${ }^{3}$ \\ 1. Paediatrics, All India Institute of Medical Sciences, Mangalagiri, IND 2. Paediatrics, Jawaharlal Institute of \\ Postgraduate Medical Education and Research, Puducherry, IND 3. Biochemistry, Jawaharlal Institute of Postgraduate \\ Medical Education and Research, Puducherry, IND
}

Corresponding author: Sarthak Das, drsarthakdas2222@gmail.com

\section{Abstract \\ Introduction}

The study aims to evaluate the association between a deficiency of Vitamin D level with the duration of hospital stay, pediatric intensive care unit (PICU) stay, and ventilation; the pediatric risk of mortality (PRISM) score, and the rate of readmission.

\section{Materials and methods}

This prospective observational study was conducted from November 2014 to October 2015, and the study population consisted of children admitted to the pediatric intensive care unit (PICU) in a tertiary care hospital of Puducherry. After measuring vitamin D levels, children were allotted into three categories depending on their serum 25(OH)D levels as the sufficient group (25[OH]D level $\geqslant 30 \mathrm{ng} / \mathrm{mL}$ ), insufficient group $(25[\mathrm{OH}] \mathrm{D}$ level $=20-29.9 \mathrm{ng} / \mathrm{mL})$, and deficient group $(25[\mathrm{OH}] \mathrm{D}$ level $<20 \mathrm{ng} / \mathrm{mL})$. Among these three groups, the duration of hospital stay, PICU stay, and ventilation; the PRISM score, and the rate of readmission were compared.

\section{Results}

A total of 522 patients were included in the study. Based on their 25(OH)D level, 222 patients $(42.5 \%)$ were in the sufficient category, 153 patients (29.3\%) were in the deficient category, and 147 patients (28.2\%) were in the insufficient category. Vitamin $D$ deficiency state is not statistically significantly associated with the duration of hospital stay $(\mathrm{P}=.84)$, duration of PICU stay $(\mathrm{P}=.69)$, duration of ventilation $(\mathrm{P}=.48)$, PRISM score $(\mathrm{P}=.63)$, and rate of readmission $(\mathrm{P}=.91)$.

Received 08/21/2020

Review began 08/28/2020 Review ended 09/01/2020 Published 09/09/2020

๑) Copyright 2020

Das et al. This is an open access article distributed under the terms of the Creative Commons Attribution License CC-BY 4.0., which permits unrestricted use, distribution, and reproduction in any medium, provided the original author and source are credited.

\section{Conclusions}

Longer hospital stay, prolonged PICU stay, longer duration of ventilation, and higher PRISM III score were independent risk factors for higher mortality in the PICU. However, lower vitamin D levels are not statistically significant to predict mortality among the study population.

Categories: Emergency Medicine, Pediatrics, Infectious Disease

Keywords: children, paediatric intensive care unit (picu stay), prism score, ventilation, vitamin d deficiency

\section{Introduction}

Vitamin D has a significant part in calcium equilibrium and skeletal system development in the human body. Immune cells like B cells, $\mathrm{T}$ cells, and antigen-presenting cells have receptors for vitamin $\mathrm{D}$, so these immune cells produce vitamin D metabolite. The immune response is significantly regulated by vitamin D, and there is increased susceptibility to infection and autoimmune disease among vitamin D-deficient patients [1]. Other than classical bone manifestations, children with vitamin D deficiency (VDD) are susceptible to systemic involvement like the central nervous system, cardiovascular system, respiratory system, and immune system [2-5]. These organ systems have a key role in the process of pathogenesis, clinical features, complication, and recovery from the state of critical illness. Therefore, VDD is a crucial factor for morbidity and mortality in the pediatric intensive care unit (PICU) [6].

In some studies, VDD has a relevant association with severe illness at presentation, increased the requirement of inotropes, the requirement and duration of mechanical ventilation, the duration of hospitalization, and mortality rate [7-14]. In contrast, these associations were not found to be relevant in 
other studies [15-16]. Whether the administration of vitamin D influences the outcome of patients, is a silent observer, or is a clinical indicator of disease severity are different problematic statements found due to the lack of availability of evidence among children and limited interventional studies in India. There was no significant data associating VDD with clinical outcomes like the duration of PICU stay, duration of hospital stay, pediatric risk of mortality (PRISM) score, the requirement for ventilation, and the duration of ventilation in the Indian scenario [15-17].

Therefore, we conducted a prospective observational study to look for an association between VDD and the duration of hospital stay, the duration of PICU stay, the duration of ventilation, the PRISM score, and the rate of readmission.

\section{Materials And Methods}

We conducted this prospective observational study from November 2014 to October 2015, and the study population consisted of children admitted to the PICU of our tertiary care hospital.

All children aged between one month and 12 years who were admitted to the PICU were included in the study. All cases of rickets (either a known previous case or a case diagnosed for the first time after hospitalization) and cases who obtained vitamin D supplementation within the last 30 days of hospital admission, cases admitted in the PICU only for monitoring purposes following a procedure or surgery and during anti-snake venom administration were excluded from the study.

Cases were enrolled after obtaining informed consent from parents, caretakers, or guardians. Parents, caretakers, or guardians were interviewed regarding the detailed chief concern, duration of sun exposure by outdoor activities, and history of vitamin D supplementation of patients, and data were collected.

As early as possible (maximum limit up to 24 hours) after being admitted to the PICU, $2 \mathrm{~mL}$ of venous blood was collected for measuring 25(OH)D levels. The blood sample was allowed to clot at room temperature and centrifuged for the preparation of serum. The prepared serum sample was frozen at $-80^{\circ} \mathrm{C}$, stored, and then used for measuring the 25(OH)D level by the enzyme-linked immunosorbent assay method, which was developed by Calbiotech (El Cajon, CA) and procured by BioDiagnosis (River Falls, WI).

After collecting patient data and measuring vitamin D levels, children were separated into three categories, depending on their serum $25(\mathrm{OH}) \mathrm{D}$ levels, as the sufficient group $(25[\mathrm{OH}] \mathrm{D}$ level $\geqslant 30 \mathrm{ng} / \mathrm{mL})$, insufficient group $(25[\mathrm{OH}] \mathrm{D}$ level $=20-29.9 \mathrm{ng} / \mathrm{mL})$, and deficient group $(25[\mathrm{OH}] \mathrm{D}$ level $<20 \mathrm{ng} / \mathrm{mL})[18]$.

\section{Statistical analysis}

For a description of the data, statistical mean $( \pm \mathrm{SD})$, frequencies (number of cases), and percentages were used as per requirements. The Kolmogorov-Smirnov test was used to check the normal distribution of data. The student's t-test was used to compare the independent quantitative variables between the study groups when normally distributed. For comparison of non-normally distributed quantitative data, a Mann-Whitney U test was used. One-way analysis of variance with post-hoc Tukey's test was used to compare more than two groups. A chi-square test was used for comparing categorical data. Fisher's exact test was used for all $2 \mathrm{x}$ 2 tables.

A binary logistic regression analysis was performed to find the significant predictors of the outcome by taking survival as the dependent variable, and variables like serum $25(\mathrm{OH}) \mathrm{D}$ levels, duration of PICU stay, hospital stay, and duration of ventilation are independent risk factors for mortality.

All statistical calculations were done using the computer programs Microsoft Excel 2007 (Microsoft Corporation, Redmont, WA) and SPSS Statistics for Windows, Version 17.0 (SPSS Inc., Chicago, Il). A P-value of less than .05 was considered statistically significant.

\section{Results}

A total of 580 patients were admitted to the PICU from November 2014 to October 2015, and 522 patients met the criteria for inclusion in the study.

Two-hundred twenty-two patients (42.5\%) were in the sufficient group, 153 patients (29.3\%) in the deficient group, and 147 patients (28.2\%) in the insufficient category. A total of 435 cases were alive, and the rest ( 87 cases) were dead. Of all 87 death cases, 29 cases (33\%) belonged to the sufficient group, 25 cases (28\%) to the insufficient group, and 33 cases (37\%) to the deficient group. Of all study children, $48.9 \%$ were infants in the age group of one month to one year, of which $34.9 \%$ were deficient, $27.8 \%$ were insufficient, and $32.7 \%$ had healthy $25(\mathrm{OH}) \mathrm{D}$ values.

The mean serum 25(OH) D levels in these different categories are represented in Table 1. 


\section{Cureus}

\begin{tabular}{|c|c|c|c|}
\hline & Sufficient & Insufficient & Deficient \\
\hline Mean (ng/mL) & 39.62 (IQR; 33.80-54.28) & $24.54(\mathrm{SD} \pm 4.147)$ & $13.37(\mathrm{SD} \pm 4.8)$ \\
\hline
\end{tabular}

TABLE 1: Mean 25(OH)D levels among groups

Abbreviation: IQR, interquartile range; SD, standard deviation

In our study population, 285 cases (54.6\%) were boys and 237 (45.4\%) were girls. The most common cause of PICU admission (27.2\%) was respiratory diseases. Of the 153 cases with a deficient level of 25(OH)D, 28.1\% had a respiratory illness, $19 \%$ had central nervous system (CNS) disease, and $13.1 \%$ had cardiovascular disease (Table 2).

\begin{tabular}{|c|c|c|c|c|c|}
\hline \multirow[b]{2}{*}{ System involved } & \multicolumn{4}{|l|}{ Vitamin D Status } & \multirow[b]{2}{*}{ P-value } \\
\hline & Sufficient N (\%) & Insufficient N (\%) & Deficient N (\%) & Total N (\%) & \\
\hline Respiratory & $63(28.4)$ & 36 (24.5) & $43(28.1)$ & $142(27.2)$ & \multirow{8}{*}{0.41} \\
\hline Cardiovascular & 30 (13.5) & $21(14.3)$ & $20(13.1)$ & 71 (13.6) & \\
\hline CNS & $46(20.7)$ & 35 (23.8) & $29(19.0)$ & $110(21.1)$ & \\
\hline Gastrointestinal & $17(7.7)$ & $10(6.8)$ & $8(5.2)$ & $35(6.7)$ & \\
\hline Renal & $8(3.6)$ & $6(4.1)$ & $9(5.9)$ & $23(4.4)$ & \\
\hline Hematological & $17(7.7)$ & $7(4.8)$ & $3(2)$ & 27 (5.2) & \\
\hline Others & $40(18.0)$ & $32(21.8)$ & $41(26.8)$ & $113(21.6)$ & \\
\hline Total (\%) & $222(42.5)$ & $147(28.2)$ & $153(29.3)$ & $522(100)$ & \\
\hline
\end{tabular}

\section{TABLE 2: System-wise distribution of cases}

Abbreviation: CNS, central nervous system

The mean duration of hospital stay in the deficient group was 206.76 hours (SD, 164.11 hours). The insufficient group's mean hospital stay was 218.61 hours (SD, 245.16 hours). The sufficient 25(OH)D level group's mean hospital stay was 210.53 hours (SD, 142.02 hours). The difference in the incidence of hospital stay is not statistically significant $(\mathrm{P}=.84$; Table 3$)$. 


\section{Cureus}

\begin{tabular}{|c|c|c|c|}
\hline Variables & Group & Mean \pm SD & P-value \\
\hline \multirow{3}{*}{ Hospital stay in hours } & Normal & $210.53 \pm 142.02$ & \multirow{3}{*}{0.84} \\
\hline & Insufficient & $218.61 \pm 245.16$ & \\
\hline & Deficient & $206.76 \pm 164.11$ & \\
\hline \multirow{3}{*}{ PICU stay in hours } & Normal & $142.39 \pm 121.19$ & \multirow{3}{*}{0.69} \\
\hline & Insufficient & $155.03 \pm 235.25$ & \\
\hline & Deficient & $140.15 \pm 136.57$ & \\
\hline \multirow{3}{*}{ PRISM III score } & Normal & $15.59 \pm 7.14$ & \multirow{3}{*}{0.63} \\
\hline & Insufficient & $16.26 \pm 8.94$ & \\
\hline & Deficient & $16.29 \pm 8.67$ & \\
\hline
\end{tabular}

TABLE 3: Duration of hospital stay, duration PICU stay (measured in hours), and PRISM III score in PICU admitted children

Abbreviations: PICU, pediatric intensive care unit; PRISM, pediatric risk of mortality; SD, standard deviation

Regarding stay in the PICU, the median duration of stay in the PICU is 140.15 hours (SD, 136.57 hours) in the deficient group, 155.03 hours (SD, 235.25 hours) in the insufficient group, and 142.39 hours (SD, 121.19 hours) in the sufficient group; these differences were not statistically significant $(\mathrm{P}=.69)$.

The PRISM III score to predict the expected mortality of PICU admitted patients was 16.29 (SD, 8.67) in the 25 (OH)D deficient group, 16.26 (SD, 8.94) in the insufficient group, and 15.59 (SD, of 7.14) in the sufficient level group, which were not statistically significant $(\mathrm{P}=.634$; Table 3$)$.

Vitamin D levels and the PRISM III score were negatively correlated as measured by Pearson correlation $\mathrm{r}=-$ 0.04 , and the correlation was not statistically significant $(\mathrm{P}=.338)$.

Only 13 of 522 patients were readmitted, of which $30.8 \%$ were from the deficient group and $23 \%$ were from the insufficient group (Table 4). Together, both contributed to $53.8 \%$ of the cases readmitted and $46.2 \%$ of cases were from the sufficient group.

\begin{tabular}{|c|c|c|c|c|}
\hline \multicolumn{5}{|c|}{ 25(OH) D Status } \\
\hline Readmission & Sufficient n (\%) & Insufficient $\mathrm{n}(\%)$ & Deficient n (\%) & P-value \\
\hline No & 216 (96.8) & 144 (98.0) & 149 (97.4) & \\
\hline Yes & $6(2.7)$ & $3(2.0 \%)$ & $4(2.6)$ & .91 \\
\hline Total (\%) & $\mathrm{n}=222(42.5 \%)$ & $\mathrm{n}=147(28.2 \%)$ & $\mathrm{n}=153(29.3 \%)$ & \\
\hline \multicolumn{5}{|l|}{ Ventilation } \\
\hline No & 116 (52.3) & $70(47.6)$ & $69(45.1)$ & \\
\hline Yes & $106(47.7)$ & 77 (52.4) & $84(54.9)$ & .48 \\
\hline Total (\%) & $\mathrm{n}=222(42.5)$ & $\mathrm{n}=147(28.2)$ & $n=153(29.3)$ & \\
\hline
\end{tabular}

TABLE 4: 25(OH)D status with rate of readmission and ventilation requirement in the PICU

Abbreviation: PICU, pediatric intensive care unit.

The ventilation requirement in the PICU was higher in the 25(OH)D deficient group (54.9\%) compared with the insufficient group (52.4\%) and the sufficient group (47.7\%). There was no statistical significance in our 


\section{Cureus}

comparisons $(\mathrm{P}=.48)$. The association between vitamin $\mathrm{D}$ status with serum calcium and phosphate level is presented in Table 5 .

\begin{tabular}{|c|c|c|c|c|}
\hline \multirow[t]{2}{*}{ Variables } & \multicolumn{3}{|c|}{ Mean Vitamin D Status } & \multirow[t]{2}{*}{ P-value } \\
\hline & Sufficient ( \pm SD) & Insufficient ( \pm SD) & Deficient ( \pm SD) & \\
\hline $\mathrm{Ca}$ & $9.24 \pm 1.23$ & $10.33 \pm 9.78$ & $9.06 \pm 1.46$ & .14 \\
\hline $\mathrm{PO}_{4}$ & $4.04 \pm 0.98$ & $4.22 \pm 1.37$ & $4.13 \pm 1.01$ & .44 \\
\hline
\end{tabular}

\section{TABLE 5: Serum calcium and phosphate levels associated with 25(OH)D}

Abbreviations: $\mathrm{Ca}$, calcium, $\mathrm{PO}$, phosphate; SD, standard deviation.

In univariate analysis, the variants found to be significantly associated with mortality were selected for multivariate analysis to determine the independent predictors of mortality. In multi-logistic regression analysis, mortality was not associated with vitamin D levels, but the duration of PICU stay, hospital stay, and duration of ventilation were associated with mortality (Table 6).

\begin{tabular}{|c|c|c|c|c|}
\hline \multirow{3}{*}{ Variables } & \multicolumn{3}{|c|}{ Logistic Regression: Mortality $\left(R^{2}=0.46\right)$} & \multirow{3}{*}{ P-value } \\
\hline & \multirow{2}{*}{ Odds Ratio } & \multicolumn{2}{|c|}{ 95\% Cl for EXP(B) } & \\
\hline & & Lower & Upper & \\
\hline Vitamin D levels & 0.996 & 0.974 & 1.005 & .192 \\
\hline PICU Stay & 1.024 & 1.007 & 1.042 & $<.01$ \\
\hline Hospital Stay & 0.98 & 0.975 & 0.985 & $<.01$ \\
\hline $\mathrm{Ca}$ & 0.922 & 0.706 & 1.203 & .55 \\
\hline $\mathrm{PO}_{4}$ & 1.022 & 0.757 & 1.38 & .88 \\
\hline Duration of ventilation & 1.02 & 1.01 & 1.031 & $<.01$ \\
\hline Ventilation & 1.007 & 0.967 & 1.048 & .74 \\
\hline PRISM III score & 0.28 & 1.08 & 1.12 & $<.01$ \\
\hline
\end{tabular}

\section{TABLE 6: Multi-logistic regression for mortality}

Abbreviations: $\mathrm{Ca}$, calcium; $\mathrm{Cl}$, confidence interval; EXP(B), PICU, pediatric intensive care unit; PO4, phosphate; PRISM, pediatric risk of mortality

\section{Discussion}

The prevalence rate of VDD observed in the present study is $29.3 \%$, which is a smaller percentage than those reported by previous studies, which ranged from $30 \%$ to $85.7 \%$ [7,10,19-23]. The difference in the prevalence of VDD might be due to the duration of outdoor activity with quality of sun exposure, the exposed surface area of the skin during outdoor activity, pigmentation of the skin, nutritional status, and various genetic factors [7].

In the present study population, $48.9 \%$ were infants (i.e., aged one month to one year). The mean $25(\mathrm{OH}) \mathrm{D}$ level was lowest in the one month to one year age group despite children in this age group having the protection of breast milk feeding. This may suggest a case of pre-existing VDD in lactating mothers of study participants.

Sankar et al. found a median serum vitamin D level of $5.8 \mathrm{ng} / \mathrm{mL}$ (IQR: 4-8) in 25(OH)D deficient patients and a median 25(OH)D level of $22.5 \mathrm{ng} / \mathrm{mL}$ (IQR 16.4-31.3) [20]. The median value of serum vitamin $\mathrm{D}$ of the present study was $13.37 \mathrm{ng} / \mathrm{mL}$ (SD $\pm 4.8 \mathrm{ng} / \mathrm{mL}$ ), which was higher than the study done in Delhi. In the present study, there was a higher level of median serum vitamin $\mathrm{D}(28 \mathrm{ng} / \mathrm{mL})$ among one to 11 -year-old 
children than in a USA study [24]. The wide variation in the median level of vitamin D level in different countries is probably due to regional trends in nutritional status, sun exposure duration, skin pigmentation, climatic changes, dietary habits, and underlying medical illnesses between study populations.

In the present study, the distribution of medical involvement was similar to the distribution reported by Venkatram et al., with respiratory cases as the predominant cause of admission involving $18.8 \%$ cases, $12.5 \%$ central nervous system (CNS) cases, and $10.5 \%$ as gastrointestinal (GI) cases [25].

The mean duration of hospital stay is aligned with those reported by Amrein et al. in Austria, in 2014, in their study of critically ill adult patients who found no significant change in the duration of hospital stay between vitamin D3 supplementation and the placebo group [26]. However, a meta-analysis conducted by Zhang et al. found that the duration of hospital stay was prolonged in hypovitaminosis D in 2014 [27].

The present study group found very little difference in the mean duration of PICU stay between study groups, but there was an increased duration of PICU stay in the 25(OH)D deficient group by approximately 72 hours as compared to the sufficient group, which was conducted by another study in India [20]. The study by McNally reported a mean PICU stay of four days, which was shorter than our findings [8]. The difference in duration is probably due to individual clinical case profiles, diagnosis at the time of admission, age of the patient, the nutritional profile of the patient, and genetic factors. Also, the duration of PICU stay was associated with the duration of ventilation and severity of illness. However, in the present study, PICU severity scores and duration of ventilation were not associated with vitamin D levels; therefore, the same was also replicated in the duration of the PICU stay.

The mean duration of mechanical ventilation in our study was three days, which was similar to the 3.5-day mean reported in a Canadian study but briefer than the nine-day mean reported by a north Indian study $[1,3]$. The discrepancies may be due to adequate sunlight exposure during outdoor activity, independent of the dietary profile. Moreover, the children in our study population may have good nutritional status, which led to shorter disease duration, better recovery, and shorter duration of mechanical ventilation [1,3]. The duration of mechanical ventilation was significantly associated with mortality in the vitamin D deficient group.

The mean PRISM III score was 16.1 (SD, 8.14) in our study. Vitamin D levels and PRISM III score are negatively correlated as measured by the Pearson correlation $\mathrm{r}=-.04$ and statistically not significant $(\mathrm{P}=$ .33), which means that the lower the vitamin D levels, the higher the PRISM III score will be, and the observed mortality is the same as those reported in other studies [28]. The median PRISM-III score was 5 (IQR, 0-11.5), and it was inversely correlated with 25(OH)D level $(\mathrm{r}=20.23, \mathrm{P}<.0001)$ reported by McNally et al. [4]. In both studies, the PRISM III score was negatively correlated with 25(OH)D levels, but the score was higher in our study as compared to the results of a study done in Canada [20]. This difference is probably due to a discrepancy in the clinical status of patients, especially the vitals of the patient and the severity of disease at the time of presentation to the PICU. A similar study from northern India had reported that the PRISM score, sequential organ failure assessment score, and duration of PICU stay were not associated with 25(OH)D deficiency [15].

Lower serum 25(OH)D levels had not been consistently associated with increased mortality or predictive mortality score in children [20]. The 90-day mortality did not differ among patients with or without VDD ( $28.3 \%$ vs. $28.5 \%, \mathrm{P}=.78$ ) in a study from Finland [29].

The events of hypocalcemia were not significantly observed in patients in a VDD state in our study. The cases with at least one hypocalcemia event had characteristically low vitamin D levels than compared to cases with healthy calcium status seen in a Canadian study [4].

Longer hospital stay $(\mathrm{P} \leqslant .01)$, prolonged PICU stay $(\mathrm{P} \leqslant .01)$, longer duration of ventilation $(\mathrm{P} \leqslant .01)$, and PRISM III score $(\mathrm{P} \leqslant .01)$ were independent risk factors for higher mortality in the PICU. However, lower vitamin D levels are not statistically significant in predicting mortality in multiple logistic regression analysis $(\mathrm{P}=.19)$. Contrary to our findings, mortality was not associated with the PRISM score or prolonged PICU stay in a study reported from Haryana, India [30].

On multivariate analysis, the association between length of ICU stay and VDD remained significant, even after adjusting for key baseline variables, diagnosis, illness severity, pediatric logistic organ dysfunction, and need for fluid boluses, ventilation, inotropes, and mortality (adjusted mean difference 3.5 days; $95 \%$ CI: $0.50-6.53 ; \mathrm{P}=.02)[20]$.

Septic shock, multiorgan dysfunction syndrome, duration of mechanical ventilation, blood culture positivity, hypocalcemia, and length of PICU stay were not modified by the presence of 25(OH)D deficiency in a study from north India [24].

Hypovitaminosis D and its association on mortality and morbidity (increased length of stay, positive blood culture, duration of mechanical ventilation, infections) show a positive correlation. Even though causality 
was not established, studies conducted globally have supported this association [7,21,25].

The strengths of our study include an adequate sample size for analysis. The duration of the study was one full year, so the factors responsible for vitamin D metabolisms like climate shift and outcome of sunlight exposure were minimized. This study described the prevalence of vitamin D among critically ill children admitted to the PICU with highly deranged physiology of the vital organs. A limitation of our study was that the real picture of vitamin D prevalence at the community level could not be deduced, as the site of the study was in the PICU of a tertiary hospital.

\section{Conclusions}

Our study compared different variables like duration of PICU stay, hospital stay, culture positivity, and calcium and phosphate with vitamin D levels and analyzed to see the correlation to vitamin D status. A longer hospital stay, prolonged PICU stay, longer duration of ventilation, and a higher PRISM III score were independent risk factors for higher mortality in the PICU. However, lower vitamin D levels were not significant to predict mortality among the study population.

\section{Additional Information \\ Disclosures}

Human subjects: Consent was obtained by all participants in this study. JIPMER Ethical committee issued approval GMRC/PAED/12/2014. Animal subjects: All authors have confirmed that this study did not involve animal subjects or tissue. Conflicts of interest: In compliance with the ICMJE uniform disclosure form, all authors declare the following: Payment/services info: All authors have declared that no financial support was received from any organization for the submitted work. Financial relationships: All authors have declared that they have no financial relationships at present or within the previous three years with any organizations that might have an interest in the submitted work. Other relationships: All authors have declared that there are no other relationships or activities that could appear to have influenced the submitted work.

\section{References}

1. Aranow C: Vitamin D and the immune system. J Investig Med. 2011, 59:881-886.

2. McNally JD, Nama N, O'Hearn K, et al.: Vitamin D deficiency in critically ill children: a systematic review and meta-analysis. Crit Care. 2017, 21:287. 10.1186/s13054-017-1875-y

3. Zittermann A, Gummert JF: Nonclassical vitamin D action. Nutrients. 2010, 2:408-425. 10.3390/nu2040408

4. Kim SY: The pleiomorphic actions of vitamin D and its importance for children . Ann Pediatr Endocrinol Metab. 2013, 18:45-54. 10.6065/apem.2013.18.2.45

5. Ginde AA, Mansbach JM, Camargo CA Jr: Vitamin D, respiratory infections, and asthma . Curr Allergy Asthma Rep. 2009, 9:81-87. 10.1007/s11882-009-0012-7

6. Lee P, Nair P, Eisman JA, Center JR: Vitamin D deficiency in the intensive care unit: an invisible accomplice to morbidity and mortality?. Intensive Care Med. 2009, 35:2028-2032. 10.1007/s00134-009-1642-X

7. Madden K, Feldman HA, Smith E, et al.: Vitamin D deficiency in critically ill children . Pediatrics. 2012, 130:421-428. 10.1542/peds.2011-3328

8. McNally J, Menon K, Chakraborty P, Fisher L, Williams KA, Al-Dirbashi OY, Doherty DR: The association of vitamin D status with pediatric critical illness. Pediatrics. 2012, 130:429-436. 10.1542/peds.2011-3059

9. Rippel C, South M, Butt W, Shekerdemian LS: Vitamin D status in critically ill children . Intensive Care Med. 2012, 38:2055-2062. 10.1007/s00134-012-2718-6

10. Ebenezer K, Job V, Antonisamy B, Dawodu A, Manivachagan MN, Steinhoff M: Serum vitamin D status and outcome among critically ill children admitted to the pediatric intensive care unit in South India. Indian J Pediatr. 2016, 83:120-125. 10.1007/s12098-015-1833-0

11. Rey C, Sánchez-Arango D, López-Herce J, Martínez-Camblor P, García-Hernández I, Prieto B, Pallavicini Z: Vitamin D deficiency at pediatric intensive care admission . J Pediatr (Rio J). 2014, 90:135-142. 10.1016/j.jped.2013.08.004

12. Ayulo M Jr, Katyal Ch, Agarwal Ch, Sweberg T, Rastogi D, Markowitz D, Ushay HM: The prevalence of vitamin D deficiency and its relationship with disease severity in an urban pediatric critical care unit. Endocr Regul. 2014, 48:69-76. 10.4149/endo_2014_02_69

13. Korwutthikulrangsri M, Mahachoklertwattana P, Lertbunrian R, Chailurkit LO, Poomthavorn P: Vitamin D deficiency and adrenal function in critically ill children. J Med Assoc Thai. 2015, 98:365-372.

14. Hebbar KB, Wittkamp M, Alvarez JA, McCracken CE, Tangpricha V: Vitamin D deficiency in pediatric critical illness. J Clin Transl Endocrinol. 2014, 1:170-175. 10.1016/j.jcte.2014.09.002

15. Ponnarmeni S, Kumar AS, Singhi S, et al.: Vitamin D deficiency in critically ill children with sepsis . Paediatr Int Child Health. 2016, 36:15-21. 10.1179/2046905515Y.0000000042

16. Dayal D, Kumar S, Sachdeva N, Kumar R, Singh M, Singhi S: Fall in vitamin D levels during hospitalization in children. Int J Pediatr. 2014, 2014:291856. 10.1155/2014/291856

17. Kumar MK, Das S, Biswal N, Parameswaran N, Nanda N: Vitamin D status at admission and its association with mortality in children admitted to the pediatric intensive care unit. Cureus. 2020, 2:e8413. 10.7759/cureus.8413

18. Holick M, Binkley N, Bischoff-Ferrari H, et al.: Evaluation, treatment, and prevention of vitamin D deficiency: an Endocrine Society clinical practice guideline. J Clin Endocrinol Metab. 2011, 96:1911-1930. 10.1210/jc.2011-0385 
19. Vijayaraj V, Nanjundappa C: Serum vitamin D levels in children admitted to the pediatric intensive care unit (PICU) and its correlation with disease severity - a cross-sectional study. Pediatr Crit Care Med. 2018, 19: 9091. 10.1097/01.pcc.0000537596.10445.52

20. Sankar J, Lotha W, Ismail J, Anubhuti C, Meena RS, Sankar MJ: Vitamin D deficiency and length of pediatric intensive care unit stay: a prospective observational study. Ann Intensive Care. 2016, 6:3. 10.1186/s13613015-0102-8

21. Braun AB, Gibbons FK, Litonjua AA, Giovannucci E, Christopher KB: Low serum 25-hydroxyvitamin D at critical care initiation is associated with increased mortality. Crit Care Med. 2012, 40:63-72. 10.1097/CCM.0b013e31822d74f3

22. Zhu Z, Zhan J, Shao J, et al.: High prevalence of vitamin D deficiency among children aged 1 month to 16 years in Hangzhou, China. BMC Public Health. 2012, 12:126. 10.1186/1471-2458-12-126

23. de Haan K, Groeneveld AJ, de Geus HR, Egal M, Struijs A: Vitamin D deficiency as a risk factor for infection, sepsis and mortality in the critically ill: systematic review and meta-analysis. Crit Care. 2014, 18:660. 10.1186/s13054-014-0660-4

24. Mansbach JM, Ginde AA, Camargo CA: Serum 25-hydroxyvitamin D levels among US children aged 1 to 11 years: do children need more vitamin D?. Pediatrics. 2009, 124:1404-1410. 10.1542/peds.2008-2041

25. Venkatram S, Chilimuri S, Adrish M, Salako A, Patel M, Diaz-Fuentes G: Vitamin D deficiency is associated with mortality in the medical intensive care unit. Crit Care. 2011, 15:R292. 10.1186/cc10585

26. Amrein K, Schnedl C, Holl A, et al.: Effect of high-dose vitamin D3 on hospital length of stay in critically ill patients with vitamin D deficiency. The VITdAL-ICU randomized clinical trial. JAMA. 2014, 312:1520-1530. 10.1001/jama.2014.13204

27. Zhang Y-P, Wan Y-D, Sun T-W, Kan Q-C, Wang L-X: Association between vitamin D deficiency and mortality in critically ill adult patients: a meta-analysis of cohort studies. Crit Care. 2014, 18:684. 10.1186/s13054-014-0684-9

28. Taori RN, Lahiri KR, Tullu MS: Performance of PRISM (Pediatric Risk of Mortality) score and PIM (Pediatric Index of Mortality) score in a tertiary care pediatric ICU. Indian J Pediatr. 2010, 77:267-271. 10.1007/s12098010-0031-3

29. Ala-Kokko T, Mutt S, Nisula S, et al.: Vitamin D deficiency at admission is not associated with 90-day mortality in patients with severe sepsis or septic shock: observational FINNAKI cohort study. Ann Med. 2016, 48:67-75. 10.3109/07853890.2015.1134807

30. Kaur G, Vinayak N, Mittal K, Kaushik JS, Aamir M: Clinical outcome and predictors of mortality in children with sepsis, severe sepsis, and septic shock from Rohtak, Haryana: a prospective observational study. Indian J Crit Care Med. 2014, 18:437-441. 